\title{
Assessment of the quality of stored blood for transfusion at Mbarara Regional Referral Hospital, Southwestern Uganda
}

This article was published in the following Dove Press journal: Journal of Blood Medicine

\author{
Hope Alice Mbabazi Rukundo' \\ Ivan Mugisha Taremwa ${ }^{2}$ \\ Enoch Muwanguzi ${ }^{3}$ \\ Mark Ndyomubantu ${ }^{4}$ \\ Frederick Byarugaba ${ }^{5}$ \\ Bernard Natukunda ${ }^{3}$ \\ 'Department of Physiology, Faculty of \\ Medicine, Mbarara University of Science \\ and Technology (MUST), Mbarara, \\ Uganda; ${ }^{2}$ Institute of Allied Health \\ Sciences, Clarke International University, \\ Kampala, Uganda; ${ }^{3}$ Department of \\ Medical Laboratory Science, Faculty of \\ Medicine, Mbarara University of Science \\ and Technology (MUST), Mbarara, \\ Uganda; ${ }^{4}$ Department of Science \\ Laboratory Technology, Faculty of \\ Science, Mbarara University of Science \\ and Technology (MUST), Mbarara, \\ Uganda; ${ }^{5}$ Department of Microbiology, \\ Faculty of Medicine, Mbarara University \\ of Science and Technology (MUST), \\ Mbarara, Uganda
}

\begin{abstract}
Aim and objective: To assess the quality of blood stored for transfusion at Mbarara Regional Referral Hospital (MRRH) regarding bacterial contamination, malaria infection, and laboratory audit status.
\end{abstract}

Materials and methods: Whole blood and packed red blood cells at MRRH were critically inspected for visual anomalies, and a portion of this blood was aseptically collected and analyzed for Plasmodium species and bacterial contamination using culture methods. For culture positive samples, drug susceptibility testing (DST) was done using the Kirby-Bauer disc diffusion method. An audit using Stepwise Laboratory quality Improvement Process Towards Accreditation (SLIPTA) quality checklist was conducted. The obtained data were analyzed as frequencies and proportions at $95 \%$ confidence interval (CI), and significance levels of relatedness were set at $p$-values $<0.05$.

Results: Of the 202 samples analyzed, 6 (3\%) had bacteria while 3 (1.5\%) had Plasmodium falciparum trophozoites. The bacterial isolates were Staphylococcus aureus $(\mathrm{N}=4,66.7 \%)$; Corynebacterium spp $(\mathrm{N}=1,16.7 \%)$ and Micrococcus spp $(\mathrm{N}=1,16.7 \%)$. Staphylococcus aureus showed sensitivity to chloramphenicol, oxacillin, amikacin, and gentamycin. Thirty $(14.9 \%)$ of these units had visually detectable anomalies, and the laboratory audit score was $53.8 \%$.

Conclusion: The quality of some blood stored for transfusion at MRRH was inadequate, and the laboratory quality standard based on SLIPTA was low. Based on this, it is crucial to always insist on aseptic measures at all stages (phlebotomy, processing, transporting, and blood storage) and consider more assessment of the donor risk to minimize transfusiontransmitted malaria. It is plausible to standardize the hospital blood transfusion laboratory and revive hemovigilance by the hospital transfusion committee.

Keywords: bacterial contamination, Plasmodium species, blood components, laboratory quality audit, SLIPTA

\section{Background}

Rukundo

Department of Physiology, Faculty of

Medicine, Mbarara University of Science

and Technology (MUST), P.O Box 1410,

Mbarara, Uganda

Tel +25 675 I90 8II4

Email hopembabazirukundo@gmail.com

Ivan Mugisha Taremwa

Institute of Allied Health Sciences, Clarke International University, P.O Box 7782,

Kampala, Uganda

Tel +256774346368

Email imugisha@ymail.com
For two centuries now, blood transfusion has been practiced as a lifesaving intervention, but only when it is of good quality since it has a risk of transfusiontransmitted infections (TTIs) such as viral, bacterial, and parasitic. ${ }^{1}$ While sensitive and specific screening methods which detect both viral antibodies and antigens have greatly reduced viral agents, the risk of bacterial and malarial infections remains high. ${ }^{2,3}$ Bacterial contamination of stored blood for transfusion has been associated with inadequate sterility in blood collection, processing, and storage. ${ }^{3}$ This is proven by having Staphylococcus species known to be a normal skin flora as the 
main contaminants. Plasmodium species are also known to be dreadful and challenging TTI mostly to the neonates and pregnant women who are the leading blood recipients. $^{4,5}$ Transfusion-transmitted malaria (TTM) which was first reported in 1911 occurs as a result of transfusing any cellular blood components from an asymptomatic blood donor, and the parasites can remain viable even at low temperature of $2-6^{\circ} \mathrm{C} .^{6}$ Though Plasmodium falciparum is most common, other species (Plasmodium vivax, ovale and malariae) exist in Uganda, and the risk of TTM is unacceptably high as malaria remains prevalent. ${ }^{7,8}$ In light of this and to avert associated risks, the World Health Organization (WHO) commends that all donor blood ought to be screened for TTM particularly subSaharan Africa. ${ }^{9}$ Other studies have indicated a high burden of human immunodeficiency virus (HIV), hepatitis B virus (HBV), and hepatitis $\mathrm{C}$ virus (HCV). For example, the seroprevalence of $\mathrm{HIV}, \mathrm{HBV}, \mathrm{HCV}$, and syphilis among blood donors in Ethiopia was 3.8\%, 4.7\%, 0.7\%, and $1.3 \%$, respectively. ${ }^{10}$ In Southeast Gabon, frequency of $\mathrm{HIV}, \mathrm{HBV}, \mathrm{HCV}$, and Treponema pallidum markers among blood donors were reported at 3.1\%, 5.9\%, 6.2\%, and $3.3 \%$, respectively, ${ }^{11}$ while in Eritrea, the seroprevalence of $\mathrm{HIV}, \mathrm{HBV}, \mathrm{HCV}$, and syphilis was $0.8 \%, 5 \%$, $0.7 \%$, and $7.2 \%$, respectively. ${ }^{12}$ At the central blood bank of Cameroon, the HBV, HIV, and HCV infection prevalence were $12.14 \%, 4.44 \%$, and $1.44 \%$, respectively;13 whereas in Mexico, the prevalence of $\mathrm{HCV}, \mathrm{HBV}$, and HIV was $1.14 \%, 1.12 \%$, and $0.24 \%$, respectively. ${ }^{14}$ Due to improved and rapid laboratory testing, there has been mandatory testing of all donor blood for HBV, HIV, and Treponema species in most setting; ${ }^{1}$ however, bacterial contaminations of blood remain the most infectious transfusion transmission risk and has become an increasing public concern and attention. ${ }^{5}$ Data from published studies have reported a high risk of transfusion-associated bacterial contamination; for example, a study conducted among 80 refrigerated blood units in Ghana found a $17.5 \%$ contamination with bacteria. ${ }^{15}$ In Ethiopia, bacterial contamination was reported at $15.33 \%$ and $12.5 \%,{ }^{16,17}$ and $3.5 \%$ in Mbarara Regional Blood Bank, Uganda. ${ }^{3}$ These studies affirm a high burden and risk of unsafe donor blood; however, there are no novel approaches and improvements in the existing practices to limit and avert the associated risk in developing settings. At Mbarara Regional Blood Bank (MRBB), supplier of blood for transfusion to MRRH, screening for malaria parasites is not done. The standard operating procedures (SOPs) set by Uganda
Blood Transfusion Service (UBTS) such as regular blood culturing and laboratory audit were not adhered to. Yet it is also a known fact that the storage environment and handling procedures affect the blood quality. ${ }^{4}$ This study assessed the quality of blood stored for transfusion in regard to bacterial contamination and Plasmodium parasitic infection risk, visual anomalies, and a laboratory quality audit status at MRRH, Southwestern Uganda.

\section{Materials and methods}

\section{Study design, site, and duration}

A laboratory-based cross-sectional study was conducted at MRRH transfusion laboratory from January to June 2017. MRRH is located in Mbarara Municipality, Southwestern Uganda. It receives both out- and inpatients, and has a bed capacity of 400 admissions. The hospital has a transfusion laboratory locally known as "Emergency laboratory" dedicated for blood transfusion procedures. It receives blood that is already screened for TTIs from MRBB, located about $2 \mathrm{~km}$ off Mbarara-Ishaka highway in Kamukuzi division, Mbarara Municipality. MRBB is part of UBTS, located in Mbarara Municipality, Southwestern Uganda. Blood is collected from healthy voluntary non-remunerated blood donors. Blood and component issuance from the MRBB and Mbarara regional referral hospital blood bank was based on the first in, first out (FIFO) principle. This principle is utilized in the blood bank while processing and issuing blood units. By this, the first units of blood to be received from MRBB were issued first to the matched recipients. In this way, the first received had a short shelf-life, and thus were used before they expired. Upon request from MRBB, the hospital transfusion laboratory stores this blood suitably, and issues it out on the request of the hospital wards. Currently, this laboratory carries out an average of 20 transfusions daily.

\section{Sample size estimation, sampling, and recruitment criteria}

The sample size was estimated using Kish Leslie's formula. ${ }^{18}$ Given $\mathrm{n}$ as the sample size; $p$ as the prevalence of TTM reported at $13.7 \%,{ }^{19} \mathrm{~d}$ as the acceptable error $(0.05)$, and $10 \%$ proportion of attrition, a sample size of 202 blood samples was considered. The study used systematic sampling, whereby every 5 th unit of blood to be issued out was considered. The study enrolled whole and packed red blood cell units that had been tested for TTIs of HBV, HIV, and syphilis using the WHO model for blood donor screening and 
confirmatory testing (Figure 1). ${ }^{9}$ The study excluded blood units that were returned for any reason, platelet concentrates that were obtained on special request and issued to the wards immediately, and other emergencies.

\section{Visual inspection}

Blood in the bag was first inspected for collection and expiry dates, color of red blood cells, and plasma. Any abnormal inclusion in the bag and tubing was noted.

\section{Malaria testing}

Thick and thin blood smears were made from the blood remaining in the syringe after innoculating on Brain Heart Infusion. These were fixed using absolute methanol for 3 minutes, stained with $10 \%$ Giemsa for 10 minutes, airdried, and examined for malaria parasites. Parasites identification was based on its infective features and stage of maturation. The quality of the Giemsa stain was ensured by including both a negative and positive control blood smears. The examination was independently done by two members of the research team, who were trained prior to the beginning of the study. In case of a discrepancy, a third read was performed by a proficient laboratory technologist attached to a research center that is involved in malaria studies.

\section{Sample collection procedure}

Blood was then mixed and allowed to seep into the tubing. Part of the tubing was well swabbed with $70 \%$ ethanol, and $6 \mathrm{~mL}$ of blood was collected using a $10-\mathrm{mL}$ sterile syringe. Two seals were made in the tubing, one before and another after the puncture so that air could not flow into the blood bag. The blood unit sampled was re-labeled with its original donor unit number, $\mathrm{ABO}$ Rh D status, and the expiry date was reduced to within $24 \mathrm{hrs}$.

\section{Sample analysis for bacterial testing/ contamination and quality control}

Aseptically, $2.5 \mathrm{~mL}$ of blood was transferred into each of the $10 \mathrm{~mL}$ Brain Heart Infusion (BHI) broth. The inoculated BHI bottles were incubated at $37^{\circ} \mathrm{C}$ for up to 7 days while observing for microbial growth as hemolysis, turbidity, or pellicle formation. Then, a subculture on solid media, namely: Blood agar, Chocolate Agar, and MacConkey agar (Oxoid Ltd, Basingstoke, UK) was done. After 7 days of incubation in

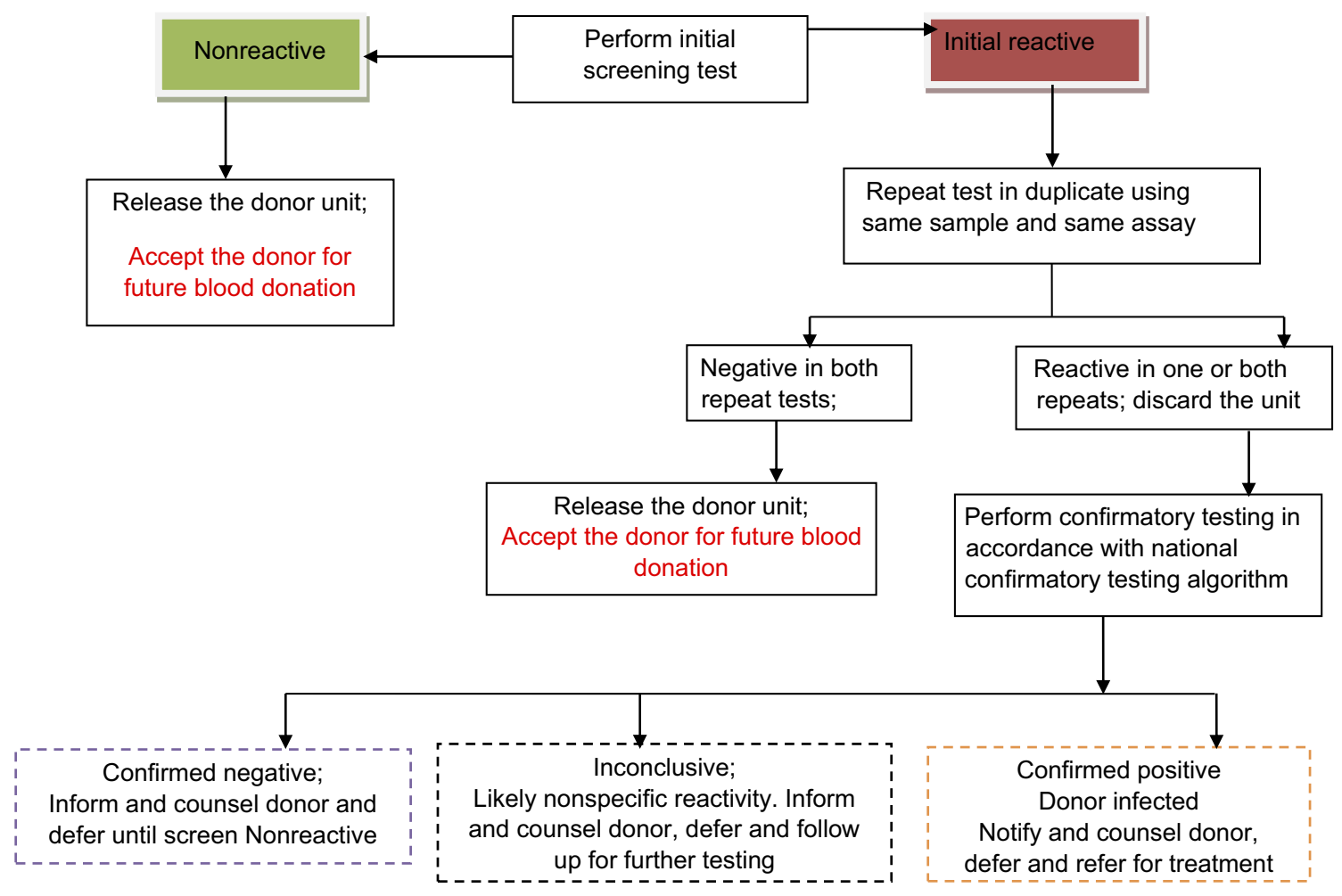

Figure I The WHO model algorithm for blood donor screening and confirmatory testing.

Notes: Adapted with permission from: World Health Organization. Screening donated blood for transfusion-transmissible infections: recommendations. 2010 ; page 52 ; Figure 2. Available from: https://www.who.int/bloodsafety/ScreeningDonatedBloodforTransfusion.pdf. Accessed May 6, 2019.9 @ World Health Organization 20I0. All rights reserved. The red text represents the deferred donor category. 
BHI, blind subculturing was done on all samples using the 3 solid culture media. Bacterial growth was identified based on colonial appearance, Gram stain reaction, and standard biochemical reactions. Antibiotic sensitivity testing on the isolates was done using Kirby-Bauer disk diffusion method, ${ }^{20}$ with antibiotic discs of; Amikacin $(30 \mu \mathrm{g})$, Ampicillin (10 $\mu \mathrm{g})$, Ceftriaxone $(30 \mu \mathrm{g})$, Chloramphenicol (30 $\mu \mathrm{g})$, Ciprofloxacin $(5 \mu \mathrm{g})$, Cotrimoxazole $(30 \mu \mathrm{g})$, Erythromycin $(5 \mu \mathrm{g})$, Gentamycin $(10 \mu \mathrm{g})$, Oxacillin $(5 \mu \mathrm{g})$, Penicillin (10 IU), and Tetracycline (10 $\mu \mathrm{g})$ (Abtek Biologicals, UK). Accurate detection of bacterial contamination was ensured by aspects included in the laboratory's routine SOPs. These included aseptic techniques at all stages of blood sample handling. Also, labeling of samples was properly done for the syringes, universal bottles, culture plates, and microscope slides. Additionally, standard strains of Staphylococcus aureus (NCTC 6571) and Escherichia coli (NCTC 10481) were set alongside as a control for Gram positive and negative organisms, respectively. Supplies of the culture media used were procured from reputable manufacturers (Becton Dickson, New Jersey, USA, and Mast Group Limited). Microbiological assays were conducted within microbiology laboratory at Mbarara University of Science and Technology, which participates in external proficiency by UKNEQAS, and strict adherence to SOPs was ensured. Regular consultations from a senior microbiologist throughout the analytical procedures were ensured.

\section{Conduct of the blood bank audit}

An audit of the hospital transfusion laboratory was carried out using the Stepwise Laboratory quality Improvement Process Towards Accreditation (SLIPTA) checklist version 2: $2015 .^{21}$ The audit was carried out by the first (HAMR) and second (IMT) authors who are experienced in using the SLIPTA checklist. This checklist consists of 12 sections of quality system essentials (QSE), comprising 110 questions worth 258 points. They are identification of non-conformities, corrective and preventive actions, evaluation and audits, facilities and biosafety, process control, equipment, organization and personnel, client management and customer service, management reviews, occurrence/indirect management and process, documents and records, information management, purchasing, and inventory. The team scheduled for internal tasks to liaise with key personnel of the laboratory and prepared the required paperwork. A careful selection of the checklist questions for the different personnel was in accordance with the hierarchy. Responses to the checklist were obtained from the laboratory technician, quality manager, and head of clinical laboratories at MRRH. They documented the results of the 12 QSE based on either, "yes", "partial", or "no". Items marked "yes" received the corresponding point value $(2$, 3 , or 5 points) depending on the relative importance of the quality essential and all elements of a question had to be present. Items marked "partial" received only 1 point, and those marked "no" scored zero. A "yes" score was given when all elements of a question were present for a given item, and thus award the corresponding points; otherwise, if any item was missing, a "partial" score was awarded. A "no" score meant that there was no single element of the question present. The score was rated according to the star rates based on scores of zero to a five-star SLIPTA grades, where 0 star ( $0-142$ points; <55\%), 1 star (143-165 points; $<55-64 \%$ ), 2 stars (166-191 points; 65-74\%), 3 stars (192217 points; 75-84\%), 4 stars (218-243 points; 85-94\%), and 5 stars $(244-258 ; \geq 95 \%) .^{21}$

The credibility of the laboratory audit was ensured by critical observations, reviews, asking open-ended questions, talking to clients, and specimen follow-up through laboratory procedures. Also, the first and second authors are trained and have used the SLIPTA checklist before, they took the lead.

\section{Quality control}

The credibility of the assays and checks was upheld by incorporating crucial aspects. First, a careful visual inspection of blood in the bag was done, and a laboratory technician attached to this section was also asked to give his/her opinion and a joint conclusion was reached upon. The expiry date was cross-checked by the technician to check consistency with the research team data.

\section{Statistical analysis}

Data were entered using Microsoft Excel 2007, cleaned, and analyzed using STATA version 13.0. Frequencies and proportions at $95 \%$ confidence interval (CI), standard deviation (SD), and means were used. Mean days of blood storage were compared with bacterial contamination and TTM risk using the Student's $t$-test. Significance levels of relatedness were set at $p$-values $<0.05$. Baseline laboratory audits and blood anomalies were presented in tables.

\section{Ethical approval}

This research was reviewed and approved by the Mbarara University of Scienceand Technology (MUST) Faculty 
Research Committee and obtained a waiver of consent from the MUST Research and Ethics Committee to use the already collected blood specimens.

\section{Results}

The study analyzed 202 blood samples. Of these, $48.5 \%$ belonged to blood group $\mathrm{O}$, and majority $(98 \%)$ were $\mathrm{Rh}$ (D) positive. The baseline characteristics are shown in Table 1.

Of the 202 blood units visually inspected, 30 (14.9\%) had visual anomalies and the majority were as a result of failure to observe the FIFO principle. In regard to the anomalies found in blood for transfusion, the small clots were in 10 units (33.3\%), mild hemolysis was seen among 2 units $(6.7 \%)$, under segmented tubing was found in 5 units $(16.7 \%)$ and there were $13(43.3 \%)$ circumstances were FIFO principle was not adhered to.

There were 3 samples (1.5\%) infected with Plasmodium falciparum trophozoites. Further, 6 samples (3\%) were contaminated with bacteria, with the majority of contaminating bacteria being Staphylococcus aureus $(\mathrm{N}=4,66.6 \%)$ then Corynebacterium $\operatorname{spp}(\mathrm{N}=1,16.7 \%)$, and Micrococcus spp $(\mathrm{N}=1,16.7 \%)$. More whole blood $(\mathrm{N}=5,83.3 \%)$ were contaminated than packed red blood cells $(\mathrm{N}=1,16.7 \%)$. There was no statistical significance between bacterial contamination and blood components $(p=0.7)$, storage duration at MRRH $(p=0.5)$, or since donation $(p=0.2)$. The drug susceptibility testing of the isolated Staphylococcus aureus indicated $100 \%$ sensitivity to Amikacin, Chloramphenicol, Oxacillin, and Gentamycin.
However, there was $100 \%$ resistance to Erythromycin, Ceftriaxone, Tetracycline, Ampicillin, Ciprofloxacin, and Cotrimoxazole. Sensitivity to Penicillin was seen among three of the four Staphylococcus aureus isolated. Corynebacterium spp showed total sensitivity to Ampicillin, Amikacin, Ciproflaxin, Cotrimoxazole, Penicillin, Gentamycin, Tetracycline, Erythromycin, and Chloramphenicol, and intermediate resistance to Ceftriaxone. Micrococcus spp showed 100\% sensitivity to Ampicillin, Amikacin, Ceftriaxone, Gentamycin, Oxacillin, Penicillin, and Tetracycline, and 100\% resistance to Ciprofloxacin and Cotrimoxazole. There was also intermediate resistance to Erythromycin and Chloramphenicol. Majority of the blood components contaminated with bacteria were whole blood 5 units $(83.3 \%)$, and only 1 unit (16.7\%) was of packed red blood cells. The bacterial contamination of blood according to ABO grouping was group A had 1 contaminated unit (16.7\%), group B had 2 contaminated units (33.3\%), and group O had 3 contaminated units $(50.0 \%)$.

The SLIPTA audit score was $53.8 \%$, which was below the minimum for a SLIPTA star (that corresponds to 55$64 \%$ ). The assessed laboratory scored highly in areas of purchasing and records, document and records plus information management. A low score was obtained in equipment, evaluation and audits, process control together with Identification of non-conformities, facilities, and biosafety. Under laboratory equipment, the notable shortfalls were poor equipment management and maintainance of equipment records. Also, the assessed laboratory did not capture

Table I Baseline characteristics of stored blood for transfusion

\begin{tabular}{|l|l|l|}
\hline Characteristic & Number & Percentage \\
\hline ABO blood group & 56 & 27.7 \\
A & 39 & 19.3 \\
B & 9 & 4.5 \\
AB & 98 & 48.5 \\
O & 198 & 98.1 \\
\hline Rh (D)positive & 4 & 1.9 \\
Rh (D) negative & & \\
\hline $\begin{array}{l}\text { Type of blood component } \\
\text { Whole blood }\end{array}$ & 143 & 70.8 \\
Packed red blood cells & 59 & 29.2 \\
\hline Mean days spent at MRRH (SD), $p=0.521)$ & $1.2(0.8)$ & \\
\hline $\begin{array}{l}\text { Mean days from donation to } \\
\text { transfusion (SD), } p=0.230)\end{array}$ & $18.7(9.0)$ & \\
\hline
\end{tabular}


the minimum information on equipment label and did not have a clear documentation plan on how to manage defective and obsolete equipment. In terms of evaluation and audits, the assessed laboratory did not have an internal audit plan and therefore lacked an action plan for improvement of laboratory quality standard. Further, the process control had shortfall as some blood request forms were inappropriate. The requesting clinician often lacked the approved requisition forms and wrote on a plain piece paper and there was a lot of missing information. Also, there was no single documentation of a non-conformance, despite the laboratory technician on duty highlighting a number of them. As such, there were no corrective actions instituted, and consequently, such or related errors and incidences continue to occur. In regard to facilities and biosafety, this laboratory lacked an evaluation report on the sufficiency of its space and experienced regular power outage, and laboratory access was almost unrestricted. The blood storage refrigerators also were not lockable with a possibility of being accessed by unauthorized personnel. First aid kits and eyewash stations were not present in the laboratory. Though the staff were keen at putting on safety gear such as laboratory coats and covering shoes, supplies of basics like hand gloves were inadequate. The respective scores for each of the 12 QSE are summarized in Table 2.

\section{Discussion}

Thirty samples (14.9\%) had anomalies that included mild hemolysis which were most likely due to lack of the right storage temperature either in the field blood donor sessions, in transportation, or in the fridges. Another anomaly was the presence of small clots in the blood, most likely as a result of not properly mixing blood in the bag within the first 3 minutes of collection. Failure to issue out blood in the order that it is received (FIFO) is an anomaly likely to bring about unnecessary blood expiry. These are concerns that ought to be addressed to alleviate the quality standards of MRRH transfusion laboratory.

The prevalence of Plasmodium species was $1.5 \%$ $(\mathrm{N}=3)$, and these were in trophozoites (ring) stage. This is lower than what was earlier reported, like $6.5 \%$ in Sudan and $7.41 \%$ in Nigeria. ${ }^{22,23}$ It was however about 2 times higher than that in Bangladesh where it was found to be $0.7 \%{ }^{24}$ The source was undoubtedly asymptomatic donors, more so as part of the study was done during the time when it was a malaria peak in the region, after the rainy season that ends around December. Malaria parasites' survival during storage at low refrigeration temperatures is well supported by the Dextrose found in the citrate phosphate dextrose adenine anticoagulant used to preserve blood in the blood bags. ${ }^{25}$

Bacterial contamination rate was found at 3\%, a figure that was slightly higher than $2.8 \%$ reported among donors in Tanzania. ${ }^{26}$ On the other hand, the contamination was lower than that reported from Ghana (between 9\% and $17.5 \%),{ }^{27} 12.5 \%$ and $15.33 \%$ in Ethiopia, ${ }^{16,17}$ and $3.5 \%$ at $\mathrm{MRBB} .^{3}$ Although intricate, the improved phlebotomy procedure of using a sample diversion pouch in current use

Table 2 MRRH transfusion laboratory audit results

\begin{tabular}{|l|l|l|l|}
\hline No* & Section & $\begin{array}{l}\text { Expected score based on SLIPTA } \\
\text { checklist score guide }\end{array}$ & Actual score (\%) \\
\hline 1 & $\begin{array}{l}\text { Identification of nonconformities, corrective and } \\
\text { preventive actions }\end{array}$ & 19 & $4(21.1)$ \\
2 & Evaluation and audits & 15 & $5(33.3)$ \\
3 & Facilities and biosafety & 43 & $19(44.2)$ \\
4 & Process control & 32 & $15(46.9)$ \\
5 & Equipment & 35 & $17(48.6)$ \\
6 & Organization and personnel & 22 & $12(54.6)$ \\
7 & Client management and customer service & 10 & $6(60.0)$ \\
8 & Management reviews & 14 & $9(64.3)$ \\
9 & Occurrence/indirect management and process improvement & 12 & $8(66.7)$ \\
10 & Documents \& records & 28 & $20(71.4)$ \\
11 & Information management & 21 & $15(71.4)$ \\
12 & Purchasing and inventory & 24 & $18(75.0)$ \\
\hline Total & & 275 & $148(53.8)$ \\
\hline
\end{tabular}

Note: *The numbers represent the order of the 12 Quality System Essentials of SLIPTA checklist.

Abbreviation: SLIPTA, Stepwise Laboratory quality Improvement Process Towards Accreditation. 
at MRBB, whereby the first $10-20 \mathrm{~mL}$ of the donated blood is not allowed to move into the main blood bag is the most likely reason for this reduction in bacterial contamination. Again, unlike in some West African countries where family replacement and commercial blood donors are the majority, ${ }^{28}$ UBTS only accepts blood from voluntary non-remunerated donors who pose a lower risk of TTIs.

Five units of whole blood (83.3\%) were contaminated with bacteria, a finding that is in agreement with previous studies done in Ghana and Nigeria, ${ }^{27}$ though it negates an earlier report in Southwestern Uganda that reported more bacterial contamination in packed red blood cells. ${ }^{3}$ There was no statistical significance between bacterial contamination and blood components $(p=0.7)$, number of days that the blood was stored at MRRH $(p=0.5)$, or number of days the blood spent in storage since donation $(p=0.2)$. Fifty percent of the contaminated blood units were of group $\mathrm{O}$, a similar pattern as earlier seen in previous studies in Ethiopia and Nigeria, ${ }^{28,29}$ though there was no significant difference in contamination of blood according to blood groups $(p=0.8)$. Among the contaminated donor units, 4 (66\%) were Staphylococcus aureus. Being part of normal skin flora, these bacteria have been identified in other studies, ${ }^{3,26,30}$ and this is attributed to poor disinfection of phlebotomy site particularly in scarred sites of repeat blood donors. The other two bacterial isolates were Corynebacterium spp and Micrococcus spp. The most probable sources of contamination in these were thought to have been dust, particularly in blood donation areas, carrier cool boxes, and in the laboratories. Also, there is a possibility that donor skin cleansing may not warrant complete sterility, and consequently, skin flora contaminates the donated blood. The DST pattern of the isolated organisms showed Staphylococcus aureus had a similar DST pattern, that is, sensitive to Amikacin, Gentamycin, Oxacillin, Chloramphenicol, and Penicillin, although resistant to Ampicillin, Ciproflaxin, Cotrimoxazole, Erythromycin, and Tetracycline. The fourth Staphylococcus spp isolated had similar DST pattern except that it was resistant to Penicillin. This could have been most probably from an asymptomatic blood donor, with drug-resistant bacteria. Corynebacterium spp and Micrococcus spp were sensitive to majority of the antibiotics, and actually, they are rarely pathogenic except in people with low immunity such as neonates. ${ }^{26}$

The SLIPTA audit score was $53.8 \%$, which is below the minimum (set at 55\%) for one star. The SLIPTA section with low scores such as equipment denotes that although the laboratory is at a regional referral level, its equipment did not conform to required standards. Further, broken down equipment such as blood transporting cool boxes and drainage sinks were not promptly repaired. Power backups were not in place yet at times the hospital's standby generator would fail to supply this laboratory with electricity. The pre-analytical, analytical, and post-analytical procedures were not well observed to avoid areas of potential pitfalls, for example, identification of blood units received from the supplying regional blood bank and at times blood returned from the wards were not always done in accordance to SOPs. The laboratory quality based on SLIPTA standard was low, similar to previous work,${ }^{31}$ and requires urgent laboratory mentorship.

The results of this study ought to be interpreted in light of the following shortfall: First, there was a blood crisis during the entire study period due to a reduction in the number of blood donors plus constant equipment failure at MRBB. Second, the study did not elucidate bacterial speciation, and the presence of Plasmodium species was identified microscopically, yet more sensitive diagnostic assays like polymerase chain reaction are recommended. Third, the six samples that gave positive results therefore were concluded as contaminated donor units ought to have had a second culture done preferably from the blood bag itself and confirmed positive with the same organism as the first culture. This is to rule out the possibility of contamination due to the sampling technique used. Fourth, this study did not clearly identify the stage of storage at which the indicated blood was contaminated with bacteria. Moreover, the study did not assess the procedures of donors' selection and sample collection.

\section{Conclusions}

This study has found that some of the blood stored for transfusion at MRRH was unsuitable for transfusion due to bacterial contamination, infection with Plasmodium species, and improper handling resulting into anomalies such as failure to observe the FIFO principle leading to the use of almost expired blood. The laboratory itself did not meet the minimum quality score for SLIPTA (at least one-star). Premised on this, a number of recommendations have been proposed: a) More aseptic measures are needed in areas of phlebotomy, transportation, and storage of blood while upholding the sample diversion pouch technique; b) SOPs ought to be strictly adhered to; c) as infection with Plasmodium species is prevalent in our setting, there is growing evidence of Plasmodium species among donor units, which suggests a need for more assessment of the donor risk to minimize TTM; d) Auditing of the laboratory should be done regularly, at biannual; and e) Hemovigilance by the hospital blood 
transfusion committee ought to be revived to ensure strict adherence to practices.

\section{Acknowledgments}

The authors are grateful to the Departments of Physiology, Medical Laboratory Sciences, and Medical Microbiology at MUST, the staff of Mbarara Regional Referral Hospital transfusion laboratory, and the team at Mbarara Regional Blood Bank. We acknowledge Young African Researchers' Development Community (YARDEC) for data management, and the technical support by JB Ariho and J Mwesigye. This study did not receive any funding. The Department of Physiology allowed the first author time off work during data collection, the Department of Medical Laboratory Sciences provided technical support for this work including mentorship, and the Department of Microbiology assisted in laboratory sample assays.

\section{Author contributions}

All authors contributed toward data analysis, drafting and critically revising the paper, gave final approval of the version to be published, and agree to be accountable for all aspects of the work.

\section{Disclosure}

The authors declare no conflicts of interest in this work.

\section{References}

1. Narayan S. Microbes and blood transfusion. Indian J Med Microbiol. 2001;19:119-126.

2. Hoppe PA. Interim measures for detection of bacterially contaminated red cell components. Transfusion. 1992;32:199-201.

3. Matte GBA, Bazira J, Richard A, Boum Y, Byaruhanga F. Bacterial contamination of blood and blood products at Mbarara Regional Blood Bank in Rural South Western Uganda. Adv Infect Dis. 2013;3:205209. doi:10.4236/aid.2013.33030

4. Dodd RU. Bacteria contamination and safety: experience in the United States. Transfus Clin Biol. 2003;10:6-9. doi:10.1016/S1246-7820(02) 00277-X

5. Kitchen AD, Chiodini PL. Malaria and blood transfusion. Vox Sang. 2006;70:77-84. doi:10.1111/j.1423-0410.2006.00733.x

6. Marshal CS, Unger KM, Deckard-Janalpour K. Risks of blood transfusion: challenge for the 21st century. Semin Anesth. 1998;17:195-207.

7. Uneke CJ, Ogbu O, Nwojiji V. Potential Risk of Induced malaria by blood transfusion in South-east Nigeria. Mcgill J Med. 2006;9:8-13.

8. Chauhan V, Negi RC, Verma B, Thakur S. Transfusion transmitted malaria in a Non-Endemic Area. Japi. 2009;57:653-654.

9. World Health Organization. Screening donated blood for transfusion-transmissible infections: recommendations. World Health Organization; 2010: page 52. Available from: https://www.who.int/bloodsafety/ ScreeningDonatedBloodforTransfusion.pdf. Accessed May 6, 2019.
10. Tessema B, Yismaw G, Kassu A, et al. Seroprevalence of HIV, HBV, $\mathrm{HCV}$ and syphilis infections among blood donors at Gondar University Teaching Hospital, Northwest Ethiopia: declining trends over a period of five years. BMC Infect Dis. 2010;10:111. doi:10.1186/1471-2334-10-111

11. Bisseye C, Mombo LE, Bie S, et al. Trends of blood- borne infectious diseases in a rural blood donation center of southeast Gabon (KoulaMoutou). Pan Afr Med J. 2018;31:81. doi:10.11604/ pamj.2018.31.81.16331

12. Keleta YT, Achila OO, Haile AW, et al. Seroprevalence of transfusion transmitted infections among blood donors in Gash Barka Zonal Blood Transfusion Center, Barentu, Eritrea, 2014 through 2017. BMC Hematol. 2019;19:5. doi:10.1186/s12878-019-0136-5

13. Fouelifack Ymele F, Keugoung B, Fouedjio JH, Kouam N, Mendibi S, Dongtsa Mabou J. High rates of hepatitis B and C and HIV infections among blood donors in Cameroon: a proposed blood screening algorithm for blood donors in resource-limited settings. $J$ Blood Transfusion. 2012;458372. doi:10.1155/2012/458372

14. Carreto-Velez MA, Carrada-Bravo T, Martinez-Magdaleno A. Seroprevalence of HBV, HCV, and HIV among blood donors in Irapuato, Mexico. Salud Publica Mex. 2003;45(Supp 5):S690-3.

15. Opoku-Okrah C, Feglo P, Amidu N, Dakorah MP. Bacterial contamination of donor blood at the Tamale teaching Hospital, Ghana. Afr Health Sci. 2009;9(1):13-18.

16. Wondimu H, Addis Z, Moges F, Shiferaw Y. Bacteriological safety of blood collected for Transfusion at University of Gondar Hospital Blood Bank, Northwest Ethiopia. ISRN Hematol. 2013:Article ID 308204. Available from:. doi:10.1155/2013/308204

17. Esmael A, Dagnew Z, Degu G. Bacterial contamination of stored blood ready for transfusion at a Referral Hospital in Ethiopia. J Clin Res Bioeth. 2014;5:176. doi:10.4172/2155-9627.1000176

18. Leslie K. Survey Sampling. New York: John Wiley and Sons, Inc; 1965.

19. Owusu-Ofori AK, Betson M, Parry CM, Stothard JR, Bates I. Transfusion-transmitted malaria in Ghana. Clin Infect Dis. 2013;56 (12):1735-1741. doi:10.1093/cid/cit130

20. Kirby W, Bauer AW, Sherris JC, et al. Antibiotic susceptibility testing by a standard single disk diffusion method. Am J Clin Pathol. 1966;46:493-496.

21. WHO. WHO Guide for the stepwise laboratory improvement process towards accreditation in the African region (with checklist). Available from: http://www.afro.who.int/en/clusters-a-pro grammes/hss/blood-safety-laboratories-a-health-technology/ blthighlights/3859-who-guide-for-the-stepwise-laboratoryimprovement-process-towards-accreditation-in-the-african-region with-checklist.html. Accessed May 9, 2019.

22. Mohamed S, Ali A, Gander AM, Yusuf K, Mustafa S. Screening Blood donors for malaria parasites in Sudan. Ethiopia J Health Dev. 2004;18 (2):69-73.

23. Ekwunife C, Ozumba N, Eneanya C, Nwaorgu O. Malaria infection among blood donors in Onitsha Urban, South East Nigeria. Sierra Leone J Biomed Res. 2011;3:1. doi:10.4314/sljbr.v3i1.66647

24. Hoque MM, Islam MA, Begum HA, Rahman M, Rahman SM, Al Mamun MA. Prevalence of malaria parasites among blood donors in selected hospitals of Dhaka City. J Dhaka Med Coll. 2008;17(2):9497. doi: $10.3329 /$ jdmc.v17i2.6590

25. McCullough J. Transfusion Medicine. Philadelphia: Elsevier Inc; 2005.

26. Malimu EZ. The Prevalence of Bacterial Contamination in Donated Blood in Dar-es-Salaam [master's thesis]. Dar es Salaam: Muhimbili University of Health and Allied Sciences; 2010. Available at: dspace. muhas.ac.tz:8080/xmlui/handle/123456789/638.

27. Adjei AA, Kuma GK, Tetty Y, et al. Bacterial contamination of blood components in three major blood transfusion centres in Accra, Ghana. Jpn J Infect Dis. 2009;62(4):265-269. 
28. Rahman A, Bolarinwa-Oldipo A, Abderin BW, Odetoyin B. Bacterial contamination of blood and blood components in a tertiary hospital setting in Nigeria. Int $J$ Infect Control. 2011;7(1):1-6.

29. Ahmed E, Zewdu D, Genet D. Bacterial Contamination of Stored Blood Ready for Transfusion at a Referral Hospital in Ethiopia. J Clin Res Bioeth. 2014;5:2. doi: 10.4172/2155-9627.1000176.
30. John B. Clinical Diagnosis and Management by Laboratory Methods. Philadelphia: WB. Saunders Company; 2001.

31. Taremwa IM, Ampaire L, Iramiot J, et al. The present and the future laboratory prospects: assessment of selected laboratory quality standards in Mbarara Municipality. Pan Afr Med J. 2017;28(129). doi:10.11604/PAMJ.SUPP.2017.28.1.2486

\section{Publish your work in this journal}

The Journal of Blood Medicine is an international, peer-reviewed, open access, online journal publishing laboratory, experimental and clinical aspects of all aspect pertaining to blood based medicine including but not limited to: Transfusion Medicine; Blood collection, Donor issues, Transmittable diseases, and Blood banking logistics; Immunohematology; Artificial and alternative blood based therapeutics; Hematology; Biotechnology/nanotechnology of blood related medicine; Legal aspects of blood medicine; Historical perspectives. The manuscript management system is completely online and includes a very quick and fair peer-review system. Visit http://www.dovepress.com/testimonials.php to read real quotes from published authors. 